\title{
Semi-classical collisional functions in a strongly correlated plasma
}

\author{
H. Ben Chaouacha ${ }^{1}$, N. Ben Nessib ${ }^{1}$, and S. Sahal-Bréchot ${ }^{2}$ \\ ${ }^{1}$ Groupe de Recherche en Physique Atomique et Astrophysique, Faculté des Sciences de Bizerte, 7021 Zarzouna, Tunisia \\ 2 Laboratoire d'Étude du Rayonnement et de la Matière en Astrophysique, UMR CNRS 8112 - LERMA, \\ Observatoire de Paris, Section de Meudon, 92195 Meudon Cedex, France
}

Received 18 July 2003 / Accepted 2 February 2004

\begin{abstract}
Collisions between atoms (or ions) and electrons play an important role in the interpretation of line spectra and for the modelling of stellar interiors. Plasma shielding effects due to electron and ion correlations are not negligible in the physical conditions of white dwarf atmospheres, owing to their high density. They also play a role in cool stars and for atomic transitions that are quasi-degenerate. In the standard formalism of Stark impact broadening of spectral lines and of cross sections, the electrostatic Coulomb potential is used to describe the interaction between the perturbing electrons and the emitting atom. Electronic correlations (screening effects) are usually taken into account by introducing a cut-off in the interaction when the electron-atom distance exceeds the Debye radius $R_{\mathrm{D}}$. A more consistent treatment to describe collective effects is the Debye-Hückel potential: the two-particle Coulomb field is shielded by the ensemble of the surrounding electrons. This is a good approximation only for high temperature and low density plasmas (weakly non-ideal plasmas), while for strongly nonideal plasmas, the Coulomb cut-off potential or the ion sphere potential are more appropriate. These potentials, which can be written as the Coulomb potential with two correcting terms, are widely used in the literature.

In this paper, we investigate the ion sphere model to describe the electron atom interaction in a strongly coupled plasma. New semi-classical collisional functions are derived for both the transition probability and the cross section, using the classical path approximation.
\end{abstract}

Key words. atomic processes

\section{Introduction}

Stark broadening of spectral lines is important for astrophysical modelling. It is found to be a reliable tool for understanding the characteristics of the plasma. This requires, in practice, a detailed knowledge of various processes, especially inelastic electronic collisions exciting and deexciting the level of the studied line in the plasma. The atomic processes in strongly correlated plasma have received particular attention in recent years.

In a previous work (Ben Nessib et al. 1997) analytical expressions of the impact semi-classical functions entering the expression of the electron collisional line width were calculated by considering the cut-off potential valid for correlated plasmas. It is found that the surrounding atoms and ions presenting in the plasma may have strong perturbing effects on the wave functions of the bound electrons. Thereby, they significantly affect the atomic transition probability as well as the atomic cross-section. Unfortunately, the cut-off model is not applicable to very high density plasmas. For a strongly

Send offprint requests to: S. Sahal-Bréchot, e-mail: sylvie.sahal-brechot@obspm.fr non-ideal plasma the ion sphere model is found to be more suitable (Jung \& Yoon 2000a; Salzmann \& Szichman 1987).

In this work, we derive analytic expressions of the semi-classical collisional functions using the static screening Coulomb interactions. As we are interested only in neutral atoms emitters for large impact parameters at relatively low energies (temperatures of the order of a few thousands or few ten thousands of degrees), we may neglect the plasma screening effects on the trajectory, and we may also use the semiclassical straight line trajectory. The corrections due to strong correlations plasma effects, even if they are not negligible, will be introduced rather in the transition probability calculation. In fact, dynamic screening Coulomb interactions using the plasma dielectric function in collision processes in non-ideal plasmas have been intensively investigated by several authors (Song \& Jung 2003b; Jung 1997, 2002, 2003; Kim \& Jung 2001; Jung $\&$ Yoon 2000b,c). However, the difference between the static and the dynamic plasma screening effects on the atomic excitation process is found to be significant only for relatively high energy projectiles (Jung \& Yoon 2000b). The excitation cross section including screening effects is shown to decrease as the non-ideality plasma parameter increases (Song \& Jung 2003a; 
Jung 2000). On the other hand, a hyperbolic orbit is reliable for ions emitters owing to the Coulomb ion-electron interaction. Such a trajectory would be also more reliable to describe screening effects on the perturber motion colliding with a neutral atom, especially for very low collision energies in the neighborhood of the threshold (Jung 1993, 1994). However, for the large impact parameter region, the straight line trajectory can be used even when the emitter is an ion (Jung 2000).

In this paper, we replace the cut-off model by the ion-sphere potential, and we give new analytical expressions, in the semiclassical approximation, for both the collisional functions of the transition probability and the cross-section. We consider the straight trajectory method to study the motion of the projectile electron, as a function of the impact parameter $\rho$ and of the ion sphere radius $R_{\mathrm{c}}$.

\section{Theory}

We will study in this part the total inelastic cross-sections entering the expression of the impact width of isolated (nonhydrogen) lines.

\subsection{Total inelastic cross-section and collisional impact broadening of isolated lines.}

Within the impact approximation the profile is Lorentzian for isolated lines. Overlapping lines are beyond the scope of the present study. For the line corresponding to the transition between the initial level $i$ and the final level $f$, the half halfwidth $w$ and the shift $d$ are given by Baranger's formula (1958):

$$
\begin{aligned}
w+i d= & N_{\mathrm{p}} \int_{0}^{\infty} v f(v) \mathrm{d} v \int_{0}^{\infty} 2 \pi \rho \mathrm{d} \rho \\
& \times\left\{1-\langle i|S| i\rangle\left\langle f\left|S^{-1}\right| f\right\rangle\right\}_{A V},
\end{aligned}
$$

where $N_{\mathrm{p}}$ designates the density of the perturber, $S$ the scattering matrix obtained for the atom-perturber interaction corresponding to the impact parameter $\rho$ and the relative velocity $v$, $f(v)$ the relative atom-perturber Maxwell distribution of velocities, and $\{\ldots\}_{A V}$ the angular average over the magnetic quantum numbers.

For the transition between the level $i\left(n_{i} l_{i} L_{i} S_{i} J_{i}\right)$ and $f\left(n_{f} l_{f} L_{f} S_{f} J_{f}\right)$, the total width at half intensity $W=2 w$ can be put in the form (Sahal-Bréchot 1969):

$$
W=N_{\mathrm{p}} \int_{0}^{\infty} v f(v) \mathrm{d} v \times\left(\sum_{j \neq i} \sigma_{i j}(v)+\sum_{j^{\prime} \neq f} \sigma_{f j^{\prime}}(v)+\sigma_{\mathrm{el}}\right),
$$

where $j, j$ refers to the perturbing levels.

The elastic contribution to the width $\sigma_{\mathrm{el}}$ is not relevant for the present paper. The inelastic cross-section $\sigma_{i j}(v)$ (respectively $\left.\sigma_{f j^{\prime}}(v)\right)$ are obtained by an integration over the impact parameter $\rho$ of the transition probabilities $P_{i j}(v, \rho)$ (respectively $\left.P_{f j^{\prime}}(v, \rho)\right)$ as:

$$
\sum_{j \neq i} \sigma_{i j}(v)=\pi R_{1}^{2} \sum_{j \neq i} P_{i j}\left(v, R_{1}\right)+\int_{R_{1}}^{R_{D}} 2 \pi \rho \mathrm{d} \rho \sum_{j \neq i} P_{i j}(v, \rho) .
$$

The perturbation theory used for the derivation of the S-matrix leads to a divergence in the integration over the impact parameter. Thus a lower cut-off $R_{1}$ is required. In addition, for high densities or for very small energy differences, an upper cut-off

$R_{D}=\left(\frac{k T}{4 \pi N_{\mathrm{e}} e^{2}}\right)^{\frac{1}{2}}$,

is introduced to take into account the shielding.

The expression for $P_{i j}$ (respectively $P_{f j^{\prime}}$ ) is given within the first order time-dependent perturbation theory by an average over the initial Zeeman states $M_{i}$ coupled to a sum over the final states $M_{j}$ (Griem et al. 1962).

$$
\begin{aligned}
& P_{i j}(v, \rho)=\frac{1}{2 J_{i}+1} \sum_{M_{i}, M_{j}} \frac{1}{\hbar^{2}} \\
& \quad \times\left|\int_{-\infty}^{+\infty}\left\langle n_{i} l_{i} J_{i} M_{i}|V(t)| n_{j} l_{j} J_{j} M_{j}\right\rangle \exp \left(\frac{\mathrm{i}\left(E_{j}-E_{i}\right) t}{\hbar}\right) \mathrm{d} t\right|^{2},
\end{aligned}
$$

where $V(t)$ designates the interaction potential between the atom and the charged perturber moving along a classical path at time $t$, and $E_{i}, E_{j}$ are the energies of the initial and final levels respectively

The cross-section $\sigma$ follows by an integration over the impact parameter $\rho$ :

$\sigma_{i j}(v)=2 \pi \int_{0}^{\infty} P_{i j}(v, \rho) \rho \mathrm{d} \rho$.

\subsection{Non-correlated semi-classical collisional functions for the total cross-section}

In a non-correlated plasma the interaction is described by the electrostatic Coulomb potential, which is expressed as:

$V=\frac{Z Z_{\mathrm{p}} e^{2}}{r_{\mathrm{p}}}-Z_{\mathrm{p}} e^{2} \sum_{i=1}^{N} \frac{1}{r_{i \mathrm{p}}}$,

where $Z e$ and $Z_{\mathrm{p}} e$ are the charges of the interacting particles, $r$, $r_{i \mathrm{p}}$ are the coordinates of the projectile electron and the bound electron, respectively (Ben Nessib et al. 1997).

$\frac{1}{r_{i \mathrm{p}}}$ is expanded in multipolar components and only the long-range part is retained in the perturbation theory:

$$
\begin{aligned}
V=\frac{Z Z_{\mathrm{p}} e^{2}}{r_{\mathrm{p}}} & -\sum_{\lambda=1}^{N} \frac{4 \pi Z_{\mathrm{p}} e^{2}}{2 \lambda+1} \times \frac{1}{r_{\mathrm{p}}^{\lambda+1}} \\
& \times \sum_{\mu=-\lambda}^{+\lambda} \sum_{i=1}^{N} r_{i}^{\lambda} Y_{\lambda \mu}\left(\widehat{r_{\mathrm{p}}}\right) Y_{\lambda \mu}^{*}\left(\widehat{r_{i}}\right) .
\end{aligned}
$$

The first term in this expression is the Coulomb term (it is null for the neutral perturbers), and it does not play a role in the calculation of the inelastic cross-sections due to its spherical symmetry. For the calculation of the cross-section between the levels having dipolar electric transition, we have to retain only the dipole term $(\lambda=1)$, i.e.:

$V_{\text {dip }}=-\frac{4 \pi Z_{\mathrm{p}} e^{2}}{3} \frac{1}{r_{\mathrm{p}}^{2}} \sum_{\mu=0, \pm 1} Y_{1 \mu}\left(\widehat{r_{\mathrm{p}}}\right) \times \sum_{i=1}^{N} r_{i} \times Y_{1 \mu}^{*}\left(\widehat{r_{i}}\right)$. 
Here, we shall adopt the usual semi-classical description of the collision process. The projectile moves along a straight path with a velocity $v$ and the radiator is localized at the origin. The impact parameter is $\rho$, and $t(=0)$ is the time of the closest approach. The transition probability from an initial state $i$ to a final state $j$ from the first order perturbation theory is given by (Sahal-Bréchot 1969; Griem et al. 1962).

$P_{i j}(v, \rho)=\frac{1}{3} \frac{Z_{\mathrm{p}}^{2} e^{4}}{\hbar} \frac{4 \pi}{3} R_{\text {line }}^{2} R_{\text {mult }}^{2} l_{>} I^{2} \sum_{\mu=0 ; \pm 1}\left|J_{1 \mu}\right|^{2}$,

where

$J_{1 \mu}=\int_{-\infty}^{+\infty} \mathrm{e}^{\mathrm{i} \omega_{i j} t} \times \frac{Y_{1 \mu}\left(\widehat{r_{\mathrm{p}}}\right)}{r_{\mathrm{p}}^{2}} \mathrm{~d} t$

is the collisional term.

$R_{\text {line }}$ and $R_{\text {mult }}$ are defined in Shore \& Menzel (1968) as:

$R_{\text {line }}\left(S L J, S L^{\prime} J^{\prime}\right)=\left[(2 J+1) \times\left(2 J^{\prime}+1\right)\right]^{\frac{1}{2}}$

$$
\times\left\{\begin{array}{ccc}
J & 1 & J^{\prime} \\
L^{\prime} & S & L
\end{array}\right\},
$$

where $R_{\text {mult }}$ in the case of one electron above a closed shell is:

$$
\begin{aligned}
R_{\text {mult }}\left(L_{c} l S L, L_{c} l^{\prime} S L^{\prime}\right) & =\left[(2 L+1) \times\left(2 L^{\prime}+1\right)\right]^{\frac{1}{2}} \\
& \times\left\{\begin{array}{ccc}
L & 1 & L^{\prime} \\
l^{\prime} & L_{\mathrm{c}} & l
\end{array}\right\} .
\end{aligned}
$$

Also, the terms $l_{>}$and $\omega_{i j}$ are defined as:

$$
\begin{aligned}
& l_{>}=\max \left(l_{i}, l_{j}\right), \\
& \omega_{i j}=\frac{E_{j}-E_{i}}{\hbar},
\end{aligned}
$$

and the radial integral $I$ is defined as:

$I=\int_{0}^{\infty} R_{n_{i} l_{i}}(r) R_{n_{j} l_{j}}(r) r \mathrm{~d} r$.

If we apply the parametric representation of a straight line trajectory (Sahal-Bréchot et al. 1996):

$\left\{\begin{array}{l}r_{\mathrm{p}}=\sqrt{\rho^{2}+v^{2} t^{2}}=\frac{\rho}{\sin \left(\theta_{\mathrm{p}}\right)}, \\ x_{\mathrm{p}}=\rho \cos \left(\phi_{\mathrm{p}}\right), \\ y_{\mathrm{p}}=\rho \sin \left(\phi_{\mathrm{p}}\right), \\ z_{\mathrm{p}}=r_{\mathrm{p}} \cos \left(\theta_{\mathrm{p}}\right) .\end{array}\right.$

The $Y_{l m}$ functions can be written as:

$Y_{10}=\sqrt{\frac{3}{4 \pi}} \cos \left(\theta_{\mathrm{p}}\right)=\sqrt{\frac{3}{4 \pi}} \frac{v t}{\sqrt{\rho^{2}+v^{2} t^{2}}}$,

and

$$
\begin{aligned}
Y_{1 \pm 1} & =\mp \sqrt{\frac{3}{8 \pi}} \sin \left(\theta_{\mathrm{p}}\right) \mathrm{e}^{ \pm \mathrm{i} \phi} \\
& =\mp \sqrt{\frac{3}{8 \pi}} \frac{\rho}{\sqrt{\rho^{2}+v^{2} t^{2}}} \mathrm{e}^{ \pm \mathrm{i} \phi} .
\end{aligned}
$$

By introducing these expressions in Eq. (11) we obtain the following expressions:

$\left|J_{10}\right|=\sqrt{\frac{3}{4 \pi}} \frac{2}{\rho v} z K_{0}(z)$,

$$
\left|J_{1 \pm 1}\right|=\sqrt{\frac{3}{8 \pi}} \frac{2}{\rho v} z K_{1}(z),
$$

where $z=\rho \omega_{i j} / v$, and $K_{0}, K_{1}$ are the modified Bessel functions. By introducing these two expressions in Eq. (10), the probability transition becomes (Sahal-Bréchot 1969; Griem et al. 1962):

$$
\begin{aligned}
P_{i j}(v, \rho) & =\frac{4 I_{\mathrm{H}}^{2}}{E\left(E_{j}-E_{i}\right)} \frac{m}{m_{\mathrm{e}}} \\
& \times f\left(n_{i} l_{i} J_{i} \rightarrow n_{j} l_{j} J_{j}\right) \times \frac{a_{0}^{2}}{\rho^{2}} A(z),
\end{aligned}
$$

where $a_{0}$ designates the Bohr radius, $m$ the electron mass, $m_{\mathrm{e}}$ the reduced electron mass, $E$ the perturber energy, $f\left(n_{i} l_{i} J_{i} \rightarrow\right.$ $n_{j} l_{j} J_{j}$ ) the oscillator strength, $I_{\mathrm{H}}$ the ionization energy of hydrogen and $A(z)$ the collision function:

$A(z)=z^{2}\left[K_{0}^{2}(z)+K_{1}^{2}(z)\right]$.

This can be written also as:

$A(z)=A_{0}(z)+2 A_{ \pm}(z)$

where

$A_{0}(z)=z^{2} K_{0}^{2}(z)$

and

$A_{ \pm}(z)=\frac{1}{2} z^{2} K_{1}^{2}(z)$.

Then, the total inelastic cross-section in a non-correlated plasma $\sigma_{i j}(v)$ is obtained after integration of the transition probability over the impact parameter, where a lower cut-off $R_{1}$ is required. An upper cut-off at the Debye radius $R_{\mathrm{D}}$ is also introduced to take into account the shielding due to the ensemble of the surrounding electrons:

$$
\begin{aligned}
\sigma_{i j}(v)= & \pi R_{1}^{2} P_{i j}\left(v, R_{1}\right) \\
& +\pi a_{0}^{2} Z_{\mathrm{P}}^{2} \frac{8 I_{\mathrm{H}}^{2}}{E\left(E_{j}-E_{i}\right)} \frac{m}{m_{\mathrm{e}}} \\
& \times f\left(n_{i} l_{i} J_{i} \rightarrow n_{j} l_{j} J_{j}\right)\left[a\left(z_{1}\right)-a\left(z_{\mathrm{D}}\right)\right],
\end{aligned}
$$

where $z_{1}=\frac{R_{1} \omega_{i j}}{v}$ and $z_{\mathrm{D}}=\frac{R_{\mathrm{D}} \omega_{i j}}{v}$.

The integration of $\frac{A(z)}{z}$ over the impact parameter leads to the $a(z)$ function:

$a(z)=\int_{z}^{\infty} \frac{A\left(z^{\prime}\right)}{z^{\prime}} \mathrm{d} z^{\prime}=z K_{0}(z) K_{1}(z)$.

As for $A(z), a(z)$ can be put in the following form:

$a(z)=a_{0}(z)+2 a_{ \pm}(z)$ 
where

$a_{0}(z)=\frac{1}{2} z^{2}\left[K_{1}^{2}(z)-K_{0}^{2}(z)\right]$,

and

$a_{ \pm}(z)=\frac{1}{4}\left\{z^{2}\left[K_{0}^{2}(z)-K_{1}^{2}(z)\right]+2 z K_{0}(z) K_{1}(z)\right\}$.

\section{Strongly correlated plasma}

\subsection{The collision functions in the cut-off model}

Multiparticles correlation effects caused by simultaneous interactions of a large number of particles have been taken into account according to different criteria to introduce the cut-off in the interaction when the electron-atom distance exceeds a certain radius. The potential must depend on the plasma properties. In a high density and low temperature plasma, the cutoff potential is reliable to describe the interaction process. In this case, the impact approximation is expressed as $T N_{\mathrm{e}}^{\frac{-1}{3}}>$ $4.2 \times 10^{-3}$ where the temperature $T$ is expressed in Kelvin and the density in $\mathrm{cm}^{-3}$. The plasma screening parameter

$\gamma=2.6 \times 10^{-3} z^{\frac{5}{3}} \frac{N_{\mathrm{e}}^{\frac{1}{3}}}{T}$

must be also greater than 0.5 (Ben Nessib et al. 1997). If only the dipolar long range part of the perturbation theory is considered, the Coulomb cut-off potential, for the atom-perturber interaction, is written as follows:

$$
\left\{\begin{aligned}
V_{\mathrm{c}}(t)= & -\frac{4 \pi Z_{\mathrm{p}} e^{2}}{3} \sum_{\mu=0 ; \pm 1}\left[\frac{Y_{1 \mu}}{r_{\mathrm{p}}^{2}}-\frac{Y_{1 \mu}}{R_{\mathrm{c}} r_{\mathrm{p}}}\right] \\
& \times \sum_{i=1}^{N} r_{i} Y_{1 \mu}^{*}\left(\widehat{r_{i}}\right), \text { if } r_{\mathrm{p}}<R_{\mathrm{c}}, \\
V_{\mathrm{c}}(t)= & 0, \text { if } r_{\mathrm{p}}>R_{\mathrm{c}},
\end{aligned}\right.
$$

where

$R_{\mathrm{c}}=\left(\frac{3 Z}{4 \pi N_{\mathrm{e}}}\right)^{\frac{1}{3}}$

designates the ion sphere radius. To obtain the transition probability, the $J_{1 \mu}$ functions in Eq. (11) have to be replaced by the correlated functions $J_{1 \mu}^{c}$ given by Ben Nessib et al. (1997):

$J_{1 \mu}^{c}=\int_{-\infty}^{+\infty} \mathrm{e}^{\mathrm{i} \omega_{i j} t}\left[\frac{Y_{1 \mu}\left(\widehat{r_{\mathrm{p}}}\right)}{r_{\mathrm{p}}^{2}}-\frac{Y_{1 \mu}\left(\widehat{r_{\mathrm{p}}}\right)}{R_{\mathrm{c}} r_{\mathrm{p}}}\right] \mathrm{d} t$.

After some calculations, the preceding $A(z)$ function becomes the correlated function $A^{\mathrm{c}}(z)$ :

$A^{\mathrm{c}}(z)=A(z)-\pi \frac{z^{2}}{z_{\mathrm{c}}} \mathrm{e}^{-z}\left[K_{0}(z)+K_{1}(z)\right]+\frac{\pi^{2}}{2} \frac{z^{2}}{z_{\mathrm{c}}^{2}} \mathrm{e}^{-2 z}$,

where $z_{\mathrm{c}}=\frac{R_{\mathrm{c}} \omega_{i j}}{v}$.

The integration of $\frac{A^{\mathrm{c}}(z)}{z}$ over $z$ involves the correlated function, which is denoted by $a^{\mathrm{c}}(z)$ :

$a^{\mathrm{c}}(z)=\int_{z}^{\infty} \frac{A^{\mathrm{c}}\left(z^{\prime}\right)}{z^{\prime}} \mathrm{d} z^{\prime}$.
This leads to the following expression:

$a^{\mathrm{c}}(z)=a(z)-\pi \frac{z}{z_{\mathrm{c}}} \mathrm{e}^{-z} K_{1}(z)+\frac{\pi^{2}}{8 z_{\mathrm{c}}^{2}}(1+2 z) \mathrm{e}^{-2 z}$.

\subsection{The collision functions in the ion sphere model}

The plasma is considered to be strongly correlated if $\gamma \geq 0.5$. In this condition, we replace the cut-off potential by the ion sphere model, which seems to be a quite reliable framework. In practice it corresponds to the Coulombian potential corrected by two terms (Jung \& Yoon 2000a; Salzmann \& Szichman 1987; Gutierrez 1994):

$\left\{\begin{array}{l}V_{\mathrm{SI}}(t)=\frac{1}{r_{i \mathrm{p}}}\left(1-\frac{3}{2} \frac{r_{\mathrm{p}}}{R_{\mathrm{c}}}+\frac{r_{\mathrm{p}}^{3}}{R_{\mathrm{c}}^{3}}\right) ; r \leq R_{\mathrm{c}} \\ V_{\mathrm{SI}}(t)=0 ; r>R_{\mathrm{c}}\end{array}\right.$

As mentioned above $R_{\mathrm{c}}$ is the ion sphere radius. Accordingly, in the expression of the transition probability, the $J_{1 \mu}$ functions in Eq. (33) have to be changed by the correlated functions $J_{1 \mu}^{\mathrm{SI}}$.

$$
\begin{aligned}
& J_{1 \mu}^{\mathrm{SI}}=\int_{-\infty}^{+\infty} \mathrm{e}^{\mathrm{i} \omega_{i j} t}\left[\frac{Y_{1 \mu}\left(\widehat{r_{\mathrm{p}}}\right)}{r_{\mathrm{p}}^{2}}-\frac{3}{2} \frac{Y_{1 \mu}\left(\widehat{r_{\mathrm{p}}}\right)}{R_{\mathrm{c}} r_{\mathrm{p}}}\right. \\
& \left.+\frac{r_{\mathrm{p}} Y_{1 \mu}\left(\widehat{r_{\mathrm{p}}}\right)}{R_{\mathrm{c}}^{3}}\right] \mathrm{d} t \text {. }
\end{aligned}
$$

Thus, we obtain after integration the collision function $A^{\mathrm{SI}}(z)$ :

$A^{\mathrm{SI}}(z)=A_{0}^{\mathrm{SI}}(z)+2 A_{ \pm}^{\mathrm{SI}}(z)$,

where

$$
\begin{aligned}
A_{0}^{\mathrm{SI}}(z)= & {\left[z K_{0}(z)-\left(\frac{3}{2}\right) \frac{\pi z}{2 z_{\mathrm{c}}} \mathrm{e}^{-z}\right.} \\
& \left.+\left(\frac{z}{z_{\mathrm{c}}}\right)^{3}\left(\frac{\sin \left(x_{\mathrm{c}} z\right)}{z^{2}}-\frac{x_{\mathrm{c}} \cos \left(x_{\mathrm{c}} z\right)}{z}\right)\right]^{2},
\end{aligned}
$$

and

$A_{ \pm}^{\mathrm{SI}}(z)=\frac{1}{2}\left[z K_{1}(z)-\left(\frac{3}{2}\right) \frac{\pi z}{2 z_{\mathrm{c}}} \mathrm{e}^{-z}+\frac{z^{2}}{z_{\mathrm{c}}^{3}} \sin \left(x_{\mathrm{c}} z\right)\right]^{2}$.

Here $x_{\mathrm{c}}=\frac{v t_{\mathrm{c}}}{\rho}$, where $t_{\mathrm{c}}$ designates the time cut-off. We notice that $x_{\mathrm{c}}$ is introduced to solve the divergence problem originating from the third term of the potential expression.

To obtain the $a^{\mathrm{SI}}(z)$ function, we have to integrate $\frac{A^{\mathrm{SI}}(z)}{z}$ over $z$ :

$a^{\mathrm{SI}}(z)=\int_{z}^{\infty} \frac{A^{\mathrm{SI}}\left(z^{\prime}\right)}{z^{\prime}} \mathrm{d} z^{\prime}$.

The cross-section collisional function is obtained finally by a simple integration of Eq. (42):

$a^{\mathrm{SI}}(z)=a_{0}^{\mathrm{SI}}(z)+2 a_{ \pm}^{\mathrm{SI}}(z)$,

where

$a_{0}^{\mathrm{SI}}(z)=a_{0}(z)+\alpha_{1}(z)+\alpha_{2}(z)+\alpha_{3}(z)+\alpha_{4}(z)$. 
Here $a_{0}(z)$ designates the collisional function for the cross section corresponding to an ideal plasma. In the $\alpha_{1}(z)$ function we find the modified Bessel functions $K_{1}$ and $K_{2}$ :

$$
\begin{aligned}
\alpha_{1}(z)= & -\frac{3}{2} \pi \frac{z}{z_{\mathrm{c}}} \mathrm{e}^{-z}\left\{\left(1+\frac{z}{3}\right) K_{1}(z)-\frac{z}{3} K_{2}(z)\right\} \\
& +\left(\frac{3}{2}\right)^{2} \frac{\pi^{2}}{16} \frac{(2 z+1)}{z_{\mathrm{c}}^{2}} \mathrm{e}^{-2 z} .
\end{aligned}
$$

The $\alpha_{2}(z), \alpha_{3}(z)$ and $\alpha_{4}(z)$ are sinusoïdal functions from the third term in the potential expression, i.e.:

$$
\begin{aligned}
& \alpha_{2}(z)=-\frac{z^{2}}{4 z_{\mathrm{c}}^{6}}\left\{1+\frac{x_{\mathrm{c}} z^{2}}{2}\right\} \\
& +\frac{z}{2 x_{\mathrm{c}} z_{\mathrm{c}}^{6}}\left\{\frac{11}{4}-\frac{z^{2} x_{\mathrm{c}}^{2}}{2}\right\} \sin \left(2 x_{\mathrm{c}} z\right) \\
& +\frac{1}{2 x_{\mathrm{c}} z_{\mathrm{c}}^{6}}\left\{\frac{11}{8 x_{\mathrm{c}}}-\frac{7 z^{2} x_{\mathrm{c}}}{4}\right\} \cos \left(2 x_{\mathrm{c}} z\right) \text {. } \\
& \alpha_{3}(z)=\left\{\frac{z^{2}}{5} K_{3}(z)-\frac{4 z}{3} K_{2}(z)+z K_{0}(z)+K_{1}(z)\right\} \\
& \times \frac{2 z \sin \left(x_{\mathrm{c}} z\right)}{z_{\mathrm{c}}^{3}} \\
& -\left\{\left(2+\frac{2 z}{3}\right) K_{1}(z)+\left(\frac{z^{2}}{5}-\frac{2 z}{3}\right) K_{2}(z)\right\} \\
& \times \frac{2 z x_{\mathrm{c}} \cos \left(x_{\mathrm{c}} z\right)}{z_{\mathrm{c}}^{3}} \text {. } \\
& \alpha_{4}(z)=-\frac{3 \pi}{2 z_{\mathrm{c}}^{4}} \frac{z \mathrm{e}^{-z}}{1+x_{\mathrm{c}}^{2}}\left\{\left(1+z x_{\mathrm{c}}^{2}\right) \sin \left(x_{\mathrm{c}} z\right)\right. \\
& \left.+x_{\mathrm{c}}(1-z) \cos \left(x_{\mathrm{c}} z\right)\right\} \\
& -\frac{3 \pi}{2 z_{\mathrm{c}}^{4}} \frac{\mathrm{e}^{-z}}{\left(1+x_{\mathrm{c}}^{2}\right)^{2}} \\
& \times\left\{\left(1+x_{\mathrm{c}}^{2}(4 z-1)\right) \sin \left(x_{\mathrm{c}} z\right)\right. \\
& \left.+2 x_{\mathrm{c}}\left(1-z\left(1-x_{\mathrm{c}}^{2}\right)\right) \cos \left(x_{\mathrm{c}} z\right)\right\} \\
& +\frac{3 \pi}{2 z_{\mathrm{c}}^{4}} \frac{x_{\mathrm{c}} \mathrm{e}^{-z}}{\left(1+x_{\mathrm{c}}^{2}\right)^{3}} \\
& \times\left\{2 x_{\mathrm{c}}\left(x_{\mathrm{c}}^{2}-3\right) \sin \left(x_{\mathrm{c}} z\right)+2\left(1-3 x_{\mathrm{c}}^{2}\right) \cos \left(x_{\mathrm{c}} z\right)\right\} \text {. }
\end{aligned}
$$

The $a_{ \pm}^{\text {SI }}$ function can be written as:

$a_{ \pm}^{\mathrm{SI}}(z)=a_{ \pm}(z)+0.5\left[\beta_{1}(z)+\beta_{2}(z)+\beta_{3}(z)+\beta_{4}(z)\right]$,

where the first term $a_{ \pm}(z)$ designates the collisional function for the cross-section corresponding to an ideal plasma, $\beta_{1}(z)$ a function of the modified Bessel functions $K_{1}$ and $K_{2}$, i.e.:

$$
\begin{aligned}
\beta_{1}(z)= & \frac{\pi}{2} \frac{z^{2}}{z_{\mathrm{c}}} \mathrm{e}^{-z}\left\{K_{1}(z)-K_{2}(z)\right\} \\
& +\left(\frac{3}{2}\right)^{2} \frac{\pi^{2}}{16} \frac{(2 z+1)}{z_{\mathrm{c}}^{2}} \mathrm{e}^{-2 z} .
\end{aligned}
$$

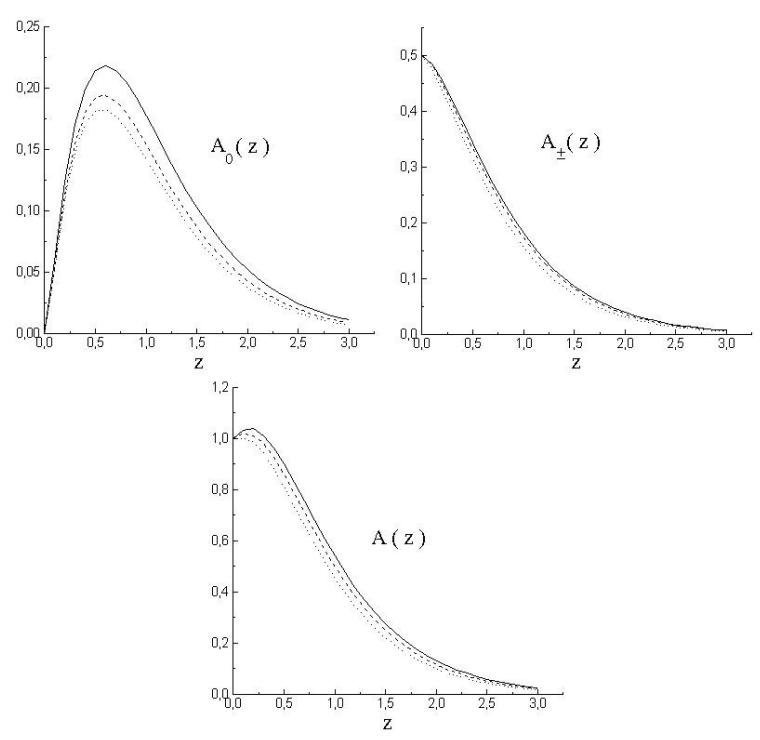

Fig. 1. Collision functions for the transition probability. Full lines: non-correlated functions $A_{0}(z), A_{ \pm}(z)$ and $A(z)$. Dotted lines: correlated functions $A_{0}^{\mathrm{c}}(z), A_{ \pm}(z)$ and $A(z)$. Dashed lines: strongly correlated functions $A_{0}^{\mathrm{SI}}(z), A_{ \pm}^{\mathrm{SI}}(z)$ and $A^{\mathrm{SI}}(z)$ for $z_{\mathrm{c}}=20$.

$\beta_{2}(z), \beta_{3}(z)$ and $\beta_{4}(z)$ are sinusoïdal functions derived from the third term in the potential expression:

$$
\begin{aligned}
\beta_{2}(z)= & \frac{1}{4 z_{\mathrm{c}}^{6}}\left\{\frac{z^{4}}{2}-\frac{z}{x_{\mathrm{c}}}\left(z^{2}-\frac{3}{2 x_{\mathrm{c}}^{2}}\right) z \sin \left(2 x_{\mathrm{c}} z\right)\right. \\
& \left.-\frac{3}{2 x_{\mathrm{c}}^{2}}\left(z^{2}-\frac{1}{2 x_{\mathrm{c}}^{2}}\right) \cos \left(2 x_{\mathrm{c}} z\right)\right\} \\
\beta_{3}(z)= & \frac{2 z^{2}}{z_{\mathrm{c}}^{3}}\left\{\left(K_{2}(z)-\frac{z}{5} K_{3}(z)\right) \sin \left(x_{c} z\right)\right. \\
& \left.-\frac{x_{\mathrm{c}} z}{5} K_{2}(z) \cos \left(x_{\mathrm{c}} z\right)\right\}, \\
\beta_{4}(z)= & -\frac{3 \pi}{2 z_{\mathrm{c}}^{4}} \frac{z^{2} \mathrm{e}^{-z}}{1+x_{\mathrm{c}}^{2}}\left\{\sin \left(x_{\mathrm{c}} z\right)+x_{\mathrm{c}} \cos \left(x_{\mathrm{c}} z\right)\right\} \\
& -\frac{3 \pi}{z_{\mathrm{c}}^{4}} \frac{z \mathrm{e}^{-z}}{\left(1+x_{\mathrm{c}}^{2}\right)^{2}}\left\{\left(1-x_{\mathrm{c}}^{2}\right) \sin \left(x_{\mathrm{c}} z\right)+2 x_{\mathrm{c}} \cos \left(x_{\mathrm{c}} z\right)\right\} \\
& +\frac{3 \pi}{z_{\mathrm{c}}^{4}} \frac{\mathrm{e}^{-z}}{\left(1+x_{\mathrm{c}}^{2}\right)^{3}}\left\{\left(3 x_{\mathrm{c}}^{2}-1\right) \sin \left(x_{\mathrm{c}} z\right)\right. \\
& \left.+x_{\mathrm{c}}\left(3-x_{\mathrm{c}}^{2}\right) \cos \left(x_{\mathrm{c}} z\right)\right\} .
\end{aligned}
$$

We have compared the effects of the Coulomb, cut-off and ion sphere potentials on the different collisional functions for a typical value $z_{\mathrm{c}}=20$. The curves relative to the transition probability functions $A_{0}(z), A_{ \pm}(z)$ and $A(z)$ are represented in Fig. 1, for these three potentials. It can be seen that both the ion sphere and cut-off models do not alter the standard functions, but they introduce a decrease in their values, especially for the lower energies. However, as expected, such a decrease seems to be more pronounced for the ion sphere model. Similar effects are observed for the three cross-section functions $a_{0}(z)$, 

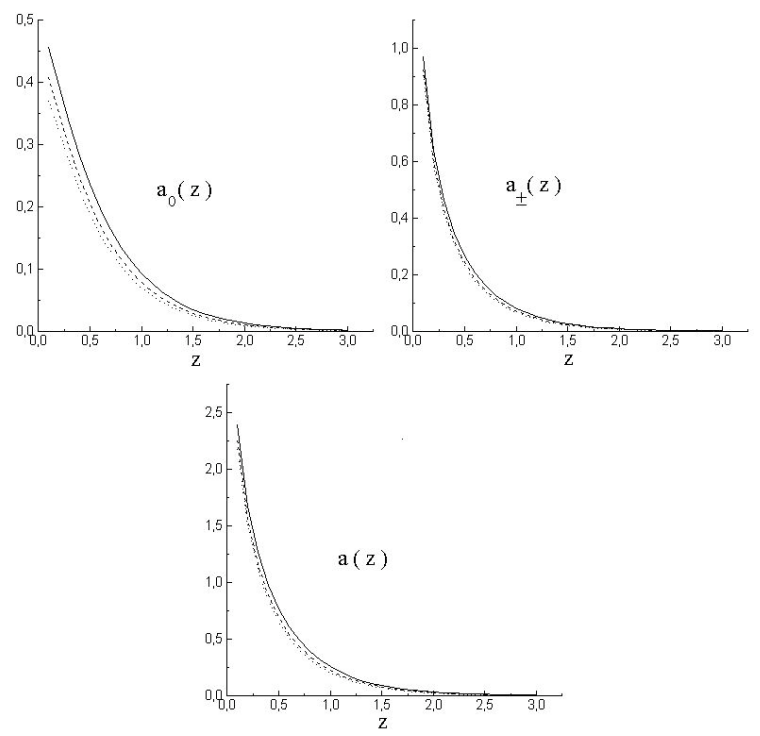

Fig. 2. Collision functions for the cross section. Full lines: noncorrelated functions $a_{0}(z), a_{ \pm}(z)$ and $a(z)$. Dotted lines: correlated functions $a_{0}^{\mathrm{c}}(z), a_{ \pm}(z)$ and $a(z)$. Dashed lines: strongly correlated functions $a_{0}^{\mathrm{SI}}(z), a_{ \pm}^{\mathrm{SI}}(z)$ and $a^{\mathrm{SI}}(z)$ for $z_{\mathrm{c}}=20$.

$a_{ \pm}(z)$, and $a(z)$, which are represented in Fig. 2 using the same value of $z_{\mathrm{c}}$.

The different corrections become insignificant as $z_{\mathrm{c}}$ increases, since the approximation of strongly coupled plasma has no sense when the $R_{\mathrm{c}}$ exceeds a certain threshold value. On the other hand, the low $z_{\mathrm{c}}$ values yield non-physical SI-curves that may exceed the Coulomb ones, which may be attributed to the fact that the $R_{\mathrm{c}}$ in this range becomes somewhat problematic.

\section{Conclusion}

The ion sphere model is expected to be well adapted to describe strongly coupled plasmas, by adding two correction terms to the standard one. We use it to calculate new semi-classical collisional functions for both the transition probability and the cross section. We have compared these collisional functions for the Coulomb, the cut-off and the ion sphere potentials. The numerical results show that the increase in the screening leads to a decrease in these functions, especially for the lower values of the impact parameter. In the next step, we will include these functions in the computer code calculating widths and shifts in the impact approximation. This will be the object of a further paper where an application to a helium line will be given.

Acknowledgements. This work has been supported by the cooperation between the French CNRS and the Tunisian DGRSRT.

\section{References}

Baranger, M. 1958, Phys. Rev., 112, 855

Ben Nessib, N., Sahal-Bréchot, S., \& Ben Lakhdar, Z. 1997, A\&A, 324, 799

Griem, H. R., Kolb, A. C., \& Oertel, G. 1962, Phys. Rev., 125, 177

Gutierrez, F. A. 1994, JQSRT, 51, 665

Jung, Y.-D. 1993, ApJ, 409, 841

Jung, Y.-D. 1994, Phys. Rev. A, 50, 3895

Jung, Y.-D. 1997, Phys. Plasmas, 4, 21

Jung, Y.-D. 2000, Eur. Phys. J. D, 11, 291

Jung, Y.-D. 2002, Phys. Plasmas, 9, 1460

Jung, Y.-D. 2003, Appl. Phys. Lett., 82, 2395

Jung, Y.-D., \& Yoon, J.-S. 2000a, ApJ, 530, 1085

Jung, Y.-D., \& Yoon, J.-S. 2000b, Phys. Scripta, 62, 46

Jung, Y.-D., \& Yoon, J.-S. 2000c, Phys. Plasmas, 7, 3917

Kim, C.-G., \& Jung, Y.-D. 2001, Phys. Scripta, 92, 335

Sahal-Bréchot, S. 1969, A\&A, 1, 91

Sahal-Bréchot, S., Vogt, E., Thoraval, S., \& Diedhiou, I. 1996, A\&A, 309, 317

Salzmann, D., \& Szichman, H. 1987, Phys. Rev. A, 35, 807

Shore, B. W., \& Menzel, D. H. 1968, Principles of Atomic Spectra (New York: Wiley and Sons)

Song, M.-Y., \& Jung, Y.-D. 2003a, J. Phys. B., 36, 2119

Song, M.-Y., \& Jung, Y.-D. 2003b, Phys. Plasmas, 10, 3051 